\title{
Recombinant Human Endostatin
}

National Cancer Institute

\section{Source}

National Cancer Institute. Recombinant Human Endostatin. NCI Thesaurus. Code C45515.

A recombinant human proteolytic fragment of the C-terminal end of type XVIII collagen.

Endostatin induces microvascular endothelial cell apoptosis and inhibits endothelial proliferation and angiogenesis, which may result in a reduction in tumor burden. This agent also may decrease hepatic metastasis by inhibiting proinflammatory cytokines and vascular cell adhesion molecule (VCAM)-dependent cell attachment to the hepatic microvasculature. $(\mathrm{NCl04})$ 